\title{
Subjectivation et désubjectivation: le cas de la violence*
}

Recebido: 04.04.14

Aprovado: 15.07 .14

\section{Michel Wieviorka**}

Resumo: Ao considerar a existência de diferentes tipos de violência e priorizando a violência coletiva, o artigo apresenta um breve exame de algumas teorias sociológicas sobre a violência, consideradas a partir de três paradigmas clássicos, aí compreendidas as análises que privilegiam a dimensão instrumental, as que veem na violência resposta ou reação a situações de crise e, finalmente, aquelas que insistem na ideia de uma cultura da violência. Os três paradigmas guardam um viés determinista, dificultando às ciências sociais melhor se capacitarem para esclarecer as condições que tornam a violência possível. $\mathrm{O}$ artigo ressalta ainda a importância de evitar o psicologismo e o sociologismo na análise da violência, apresentando duas outras orientações: na primeira, discute o interacionismo - com o componente da intersubjetividade - indicando alguns de seus aspectos positivos e, sobretudo, seus limites; na segunda, ressalta a orientação teórica centrada no sujeito, na subjetividade e, principalmente, nos processos de subjetivação e dessubjetivação. Postula a necessidade de definir os conceitos de subjetividade, sujeito, subjetivação e dessubjetivação, definindo, com esse propósito, quatro figuras de sujeito que podem até mesmo coexistir no mesmo ator. Demonstra, enfim, a insuficiência do conceito de sujeito e propõe passar, na análise, do enfoque no sujeito e na subjetividade ao enfoque nos processos de subjetivação e dessubjetivação, evitando a essencialização ou naturalização do sujeito.

Palavras-chave: violência, sujeito, subjetividade, subjetivação, dessubjetivação.

$\mathrm{L}$ a violence est un des phénomènes les plus complexes et les plus diversifiés qu'envisagent d'étudier les sciences humaines et sociales, et le terme renvoie à toute sorte d'expériences, individuelles comme collectives. Indiquons donc d'abord les limites des réflexions qui vont suivre, et précisons l'espace des conduites de violences sur lesquelles elles porteront, du moins principalement.

La violence peut être physique, morale, intellectuelle. Elle porte atteinte, ou vise à porter atteinte à l'intégrité de ceux qui en sont les victimes, et le plus souvent, tout ceci constitue un tout: blesser, tuer, violer, voler ou tenter de le faire, par exemple, c'est aussi mettre en jeu du sens, des significations, c'est affaiblir ou détruire les «capabilities» dont parle Amartya Sen ${ }^{1}$, et qui ne sont pas seulement concrètes ou physiques. Nous laisserons ici les dimensions intellectuelles et morales que charrie la violence, et en particulier nous n'entrerons pas dans la discussion qu'appelle le concept de violence symbolique, développé par Pierre Bourdieu ${ }^{2}$, et qui est assez proche de la notion d'aliénation. Pour ce sociologue, il s'agit de désigner des formes extrêmes de domination qui font que le dominé intériorise jusqu'aux catégories du dominant, et s'avère dès lors incapable de penser par lui-même, ce qui empêche la révolte ou la protestation.

\author{
* Conferência \\ proferida no \\ Departamento \\ de Sociologia da \\ Universidade de \\ Brasília em março de \\ 2014. \\ ** Diretor da \\ Fundação Maison des \\ Sciences de l'Homme \\ (FMSH), Paris, diretor \\ de estudos da École \\ des Hautes Études \\ en Sciences Sociales \\ (Ehess), pesquisador \\ e ex-diretor do \\ Centre d'Analyse \\ et d'Intervention \\ Sociologique (Cadis). \\ < Michel.Wieviorka@ \\ ehess.fr>. \\ 1. Amartya Sen, \\ Éthique et économie: \\ Paris, PUF, 2008. \\ 2. Cf., notamment, \\ Pierre Bourdieu, \\ Esquisse d'une théorie \\ de la pratique. Paris: \\ Droz, 1972.
}


La violence peut être individuelle, elle peut aussi être collective, sociale, politique notamment: c'est en priorité la violence collective qui nous occupera ici, qu'il s'agisse de révolutions, d'émeutes, ou bien encore de terrorisme. La délinquance - lorsqu'elle renvoie à des questions sociales - et le crime organisé s'inscrivent certainement aussi dans l'espace de nos analyses, et beaucoup moins le pur crime crapuleux, ou le vol, ce qu'on appelle en France le « fait divers ».

\section{Les sciences sociales et la violence}

Les théories sociologiques de la violence, ou les approches historiques, ethnologiques ou politologiques ne manquent pas, et malgré leurs grandes différences, elles ne sont pas nécessairement contradictoires. On peut en première approximation considérer qu'elles relèvent de trois grandes familles, de trois types de paradigmes. Certaines s'intéressent aux dimensions instrumentales du phénomène, aux calculs des acteurs, à leur stratégie. La violence est alors une ressource utilisée pour obtenir un résultat, c'est un moyen par rapport à des fins. L'analyse peut ici concerner aussi bien des acteurs individuels qui s'apparentent à l'homo œconomicus de la théorie économique, que des groupes et une action collective, comme dans les théories

3. Le meilleur représentant de cette orientation fut I'historien-sociologue Charles Tilly. Cf., par exemple, de cet auteur From mobilization to revolution. Reading, Mass.: Addison Wesley Publishing Co., 1978 .

4. Cf., par exemple, Ted Robert Gurr, Why men rebel. Princeton: Princeton University Press, 1970.

5. Une source importante de cette manière de penser est l'enquête de Theodor Adorno et alii. The authoritarian personality. New York: Harper, 1960. dites de la mobilisation des ressources ${ }^{3}$. Les limites de ce premier type d'approche tiennent à ce que nous enseigne l'observation historique ou empirique: la violence instrumentale est toujours susceptible de déraper, d'être emportée par d'autres logiques que purement stratégiques, d'être débordée par d'autres significations que celles relevant du calcul rationnel.

D'autres approches font de la violence une réaction à une situation, et en particulier à une situation de crise. La violence, là encore aussi bien individuelle que collective, est alors expliquée par des évènements qui la déterminent en dehors d’elle-même, par un contexte devenu insupportable, par des évolutions suscitant des frustrations devenues intolérables ${ }^{4}$. Dans cette perspective, elle est la conduite qui constitue une réponse, par exemple, aux difficultés économiques soudaines, à la menace de fermeture d'une entreprise, au chômage, à la déréliction.

Enfin, une troisième famille d'analyses, tout aussi classique, insiste sur l'idée d'une culture de la violence, qui prédisposerait les membres d'une famille, d'un groupe, d'une communauté, d'une société toute entière, plus que d'autres, à passer à la violence. II existe de nombreuses variantes de ces démarches, qui peuvent éventuellement proposer de tracer un lien entre culture et personnalité, la violence hic et nunc trouvant alors ses sources, par exemple, dans une éducation ou une socialisation primaire qui y prédisposent ${ }^{5}$. C'est ainsi que la criminologie explique parfois les crimes sexuels ou les violences sur enfants par une enfance où l'auteur a lui-même subi de telles horreurs. 
Qu'elles soient utilitaristes, portées par l'idée d'une réponse à une situation assez large, ou par celle d'une culture façonnant des prédispositions, les approches les plus classiques des sciences humaines et sociales n'échappent qu'imparfaitement au travers déterministe, qui postule une causalité, ou un faisceau de causes pour expliquer la violence. Le déterminisme est parfois poussé très loin, et de social ou culturel, il peut même dans certaines approches devenir naturel, biologique, par exemple dans les propositions contemporaines ${ }^{6}$ qui renouvellent à leur façon les vieilles théories de Lumbroso en allant chercher les sources du crime, de la violence terroriste, ou sexuelle dans le patrimoine génétique des individus - un thème journalistique autant que scientifique.

Les approches déterministes, surtout lorsqu'elles comportent des dimensions de type biologique, sont toujours suspectes aux yeux des chercheurs en sciences sociales, qui se méfient à juste titre du déterminisme, en général, et de la naturalisation des questions sociales en particulier. Elles sont de plus inquiétantes du point de vue des droits de l'homme et des valeurs démocratiques et humanistes qu'elles autorisent vite à bafouer: le détenu qui a purgé sa peine mais qui aurait un profil génétique et social jugé susceptible d'en faire un récidiviste ne doit-il pas, dans leur perspective, être maintenu en détention? Le voyageur qui désire entrer dans un pays mais dont le profil est jugé pré ou proto-terroriste ne doit-il pas être refoulé? Le passage à l'ère des " big datas », où se mêlent éventuellement données de type génétique et données sociales et culturelles est ici éminemment inquiétant pour qui se préoccupe des droits fondamentaux de la personne.

C'est pourquoi les sciences humaines et sociales, lorsqu'elles entendent résister à I'idée de causalité et au pur déterminisme, semblent plus capables d'offrir un éclairage sur les conditions qui rendent possibles la violence, que sur la violence ellemême.

L'essentiel est que les approches qui viennent d'être évoquées laissent toutes plus ou moins de côté ce qui est le plus mystérieux, et donc le plus caractéristique dans les conduites de violence: le fait que presque toujours, si elles ont une certaine importance, ou si elles se prolongent ou se répètent quelque peu, elles viennent dire autre chose que ce que suggère la théorie qui prétend les expliquer. Elles sont vite dans l'excès ou le défaut par rapport au type d'explication mobilisé. Comment se satisfaire par exemple de réduire à l'idée d'un calcul et donc d'une rationalité instrumentale une action terroriste dans laquelle des acteurs font le sacrifice de leur vie, quel calcul coût/avantages peut-on effectuer ici? Comment prétendre qu'une situation de crise explique pleinement des protestations politiques violentes lorsque surgit en leur sein la cruauté gratuite, la violence pour la violence, la violence comme fin en soi? Pourquoi certains acteurs violents éprouvent-ils le besoin de lester leurs
6. Cf., par exemple, Rose McDermott, Dustin Tingley, Jonathan Cowden, Giovanni Frazzetto \& Dominic D. P. Johnson, Monoamine oxidase A gene (MAOA) predicts behavioral aggression following provocation. Proceedings of the National Academy of Sciences, v. 106, n. 7, Fev. 2009. 
conduites de discours idéologiques, ou religieux, au point de verser parfois dans la logorrhée? Pourquoi d'autres, ou les mêmes, sont-ils sadiques?

II y a là un vaste ensemble de problèmes qu'il faut aborder de front: comment les sciences sociales peuvent-elles analyser ce qui dans les conduites de violence résiste aux approches classiques, ou les déborde, même si elles apportent aussi des éléments pertinents d'explication?

\section{Éviter les dérives psychologisantes ou sociologisantes}

Une première réponse nous déporte du côté d'une psychologisation extrême. Les conduites de violence sont parfois si déroutantes, ou si liées à des caractéristiques qui semblent hautement personnelles, que l'explication de type sociologique, politique ou ethnologique ne peut trouver qu'une place limitée. L'analyse devient alors plus volontiers psychologique, voire médicale, elle tend alors à isoler la personnalité de l'acteur, pour examiner ses antécédents, sa trajectoire, son milieu familial, et dans certains cas pour psychiatriser la violence et en faire une folie, une pathologie. Dans les cas extrêmes, une telle perspective a pour implication, ou pour conséquence de déresponsabiliser l'auteur de la violence, puisqu'il serait agi par ses pulsions, et non pas acteur.

7. Hannah Arendt, Eichmann à Jérusalem. Rapport sur la banalité du mal. Paris: Gallimard, 1966.

8. Stanley Milgram, Obedience to authority: an experimental view. London: Tavistock, 1974.
Un tel point de vue n'est pas à rejeter nécessairement, mais il n'est acceptable qu'à condition que l'on se soit bien assuré, empiriquement, qu'il en est ainsi. Le constat d'irresponsabilité ne devrait être formulé qu'une fois que l'on ait examiné en profondeur d'autres hypothèses, et certainement pas parce qu'il est invoqué par l'auteur, ou par exemple par ses avocats. Ainsi, depuis Hannah Arendt ${ }^{7}$, vivement critiquée d'ailleurs sur ce point, la thèse d'une irresponsabilité d'Adolphe Eichmann dans la disparition des Juifs d'Europe a été fréquemment avancée: il aurait agi non pas par haine des Juifs, par antisémitisme forcené, mais parce qu'il était placé dans une situation où il lui fallait obéir de façon purement administrative à une autorité légitime, un peu à la manière des étudiants dans les célèbres expériences de Stanley Milgram ${ }^{8}$, lorsqu'ils obéissent au professeur leur ordonnant de faire souffrir un cobaye répondant de travers aux questions posées.

La difficulté, avec les approches de type psychologique, est qu'elles tendent à minimiser ou à écarter les dimensions sociales, culturelles, politiques, historiques de la violence collective, et à réduire la violence individuelle au travail d'une conscience ou d'un inconscient dissocié de la société, de son évolution, des conditions qu'elle crée pour rendre ou non possible l'expression de la violence. Elles ignorent le sens de l'action, ou le séparent pour être indifférentes de la personne ou du groupe considéré. 
Pourtant, ces approches que nous appelons psychologisantes présentent aussi un intérêt essentiel: elles ont quelque chose à dire sur le passage à l'acte, sur le moment singulier ou sur les processus individuels qui y conduisent, et où s'opère l'action. II y a là un point important. Une explication purement sociologique, ou apparentée, laissera bien souvent de côté, en effet, le fait que seuls quelques individus, voire un seul, passent à l'acte, alors que des milliers, ou des centaines de milliers de personnes ont le même point de vue, les mêmes idées, partagent leurs émotions et leurs orientations, ou bien encore ont connu une trajectoire sociale comparable. La sociologie et les disciplines proches n'autorisent pas vraiment à comprendre pourquoi un acte de violence est le fait d'une personne singulière, et non pas d'autres. Illustrons cette remarque.

\section{Une illustration}

Prenons le cas de ceux qu'une littérature journalistique a parfois, depuis les attentats de Boston en 2013, appelés des «loups solitaires », terroristes agissant seuls, ou presque. En Norvège, Anton Behring Breivik a tué froidement, le 22 juillet 2011, 77 jeunes au nom d'une idéologie d'extrême droite, anti-musulmane, anti-immigrés, mâtinée de quelques références surprenantes, notamment à Israël. II a pu être arrêté par les forces de l'ordre, incarcéré et jugé. Sa défense, devant les tribunaux, a tout fait pour qu'il soit tenu comme responsable de ses actes, et donc comme ayant agi de façon politique. Le cas est singulier, exceptionnel, mais a pu trouver quelque écho ou une réelle compréhension, y compris dans son propre pays, au sein des sympathisants de l'extrême droite.

En France, Mohammed Merah, en mars 2012, a tué d'abord trois militaires, incarnation selon lui d'une armée qui agirait contre l'islam, puis trois enfants juifs et un enseignant d'une école juive de Toulouse, exprimant ainsi un antisémitisme sans limites. L'homme semble avoir agi seul, ou avec un nombre très limité de complices. La violence est un choix hautement personnel, délibéré, de façon solitaire, elle relève de l'analyse d'un individu, et pourtant, elle met en cause des affects que partage une partie de la jeunesse musulmane, en France et ailleurs - ce dont on trouve d'innombrables expressions sur la Toile ou dans les réseaux sociaux. Mais cette jeunesse, elle, ne passe pas à l'acte.

Enfin, les frères Tsamaev, en déposant des explosifs à l'arrivée du marathon de Boston, en avril 2013, ont eux aussi semble-t-il agi seuls, mais leurs attentats, même si à ce jour leur idéologie ou leur religiosité musulmane semblent floues, peuvent correspondre aux orientations d'autres personnes qu'eux-mêmes, qui peuvent s'y reconnaître - là encore, ces acteurs isolés sont connectés par internet à des réseaux planétaires. 
Dans les trois cas, le passage à l'acte est singulier, hautement personnel, mais les significations de l'acte peuvent concerner une population beaucoup plus large. C'est pourquoi l'analyse doit aller d'une perspective à une autre, de la personne individuelle au contexte socio-politique ou culturel dans lequel elle a agi.

Les significations politiques, idéologiques, éventuellement religieuses de l'acte sont ici claires, voire évidentes, et elles sont partagées par bien d'autres. Mais quand un individu seul, ou presque, passe à l'acte, une approche purement sociologique ne permet pas vraiment d'appréhender convenablement la violence. Et symétriquement, la psychologie ou la criminologie, en laissant de côté le sens mis dans leurs actes de violence par des acteurs comme ces "loups solitaires », n'est pas entièrement satisfaisante. Ils sont criminels aux yeux de la loi, ce ne sont pas pour autant nécessairement des fous, d'ailleurs, ils sont tout à fait capables d'une certaine rationalité, et ils veulent donner un sens politique, ou géopolitique, aux meurtres qu'ils ont commis.

Est-il possible de rapprocher un point de vue centré sur le sens de l'action, sa portée sociale, politique, historique, ses dimensions collectives, le souci de l'auteur d'actes violents de peser sur l'ordre du monde, d'un côté, et d'un autre côté une perspective qui s'intéresse à sa personnalité? En fait, oui, et deux orientations principales méritent ici d'être mises en discussion.

\section{Interactions}

Une première démarche est ici interactionniste, et commence en fait depuis peu à se développer à propos de la violence. Situons tout d'abord cette orientation dans son histoire récente. Celle-ci trouve son point de départ dans la grande déconstruction qui s'est opérée à partir des années 1960, quand les sciences sociales ont voulu

9. Jean-François Lyotard, La condition post-moderne. Paris: Minuit, 1979. en finir avec les " grands récits » dont parle Jean-François Lyotard", entrer dans la post-modernité, et se libérer des « grandes » théories. La recherche a pris alors ses distances avec le structuralisme, elle a cessé de laisser une place considérable au marxisme, sous ses différentes variantes. Elle a surtout délaissé le fonctionnalisme, en particulier tel qu'il était incarné par Talcott Parsons. Et dans cette évolution, des modes d'approche qui s'intéressaient à l'étude des interactions, surtout limitées, ont trouvé une nouvelle jeunesse, parfois, comme avec l'ethnométhodologie, directement dans les décombres du fonctionnalisme, mais pas nécessairement. Pour comprendre le social, le mieux, proposent ces approches, est de comprendre les méthodes par lesquelles les humains vivent ensemble, et pour cela, des observations fines, ethnographiques, sont décisives. Elles indiquent comment se développent des interactions qui, finalement, tissent la vie collective. 
Ces approches, dont la plus haute figure est certainement Erving Goffman, ont pris une assez grande distance vis-à-vis de la politique, et vis-à-vis de l'histoire. Et jusqu'à peu, elles ne s'intéressaient guère à la violence - en tout cas pas au point d'en faire un problème central. Mais en 2008, un livre de Randall Collins est venu proposer une théorisation interactionniste de la violence ${ }^{10}$ qui doit beaucoup aux perspectives ouvertes par Jack Katz dans sa sociologie des émotions ${ }^{11}$, et qui mérite toute notre attention ${ }^{12}$.

Connu jusqu'ici pour ses orientations wébériennes et sa lecture de Max Weber, Randall Collins analyse la violence dans une perspective qui devient microsociologique, et interactionniste qu'il annonçait déjà au début des années $2000^{13}$. Son approche se veut pragmatiste. De sociologique, l'analyse devient plutôt ethnologique, en tous cas elle repose sur des travaux d'ethnographie, comme ceux d'Elijah Anderson; elle doit beaucoup aussi aux travaux d'historiens tels Samuel L. A. Marshall qui s'est intéressé au comportement au combat des soldats américains pendant la Deuxième Guerre Mondiale ${ }^{14}$. Randall Collins, qui consulte avec soin des documents photographiques ou des vidéos, et qui aussi a procédé lui-même à des observations sur le terrain, considère avec minutie les expressions et les attitudes corporelles des acteurs au moment de la violence, les bruits ou les sons qu'ils émettent, le rythme des corps et les mouvements en situation, les efforts pour imposer un rythme à l'adversaire. Pour lui, la violence trouve ses causes non pas dans la subjectivité de ses protagonistes, mais dans la dynamique des situations dans lesquelles ils se trouvent.

La violence jaillit en effet, à le suivre, en situation, elle résulte, dit-il d'une " configuration émotionnelle », d'une "tension confrontationnelle » qui se dépasse ou se résout éventuellement dans la violence. Comprendre la violence, dans cette perspective, c'est donc prendre en compte ce qui se joue concrètement, lorsqu'il y a tension et, éventuellement, peur, et que certains ont recours alors, le cas échéant, à la violence. Pour Randall Collins, la « tension confrontationnelle » ne débouche que rarement sur la violence, et alors généralement partiellement seulement. Quand il y a interaction sociale immédiate, dit-il, quand les individus sont très proches les uns des autres, il est difficile d'aller au bout d'une logique de violence.

Le plus souvent, à le suivre, les situations violentes viennent prolonger des situations a-violentes, c'est-à-dire qui ne sont pas violentes en elles-mêmes. Et la violence ne s'explique pas par des motivations, être « motivé » ne suffit pas. Randall Collins voit donc dans la violence un " contournement de la tension confrontationnelle », qui repose parfois sur un rapport de force - le fort attaque le faible, le groupe attaque l'individu isolé -, sur l'attitude éventuelle du public, qui peut être un encouragement au passage à la violence, sur la mise à distance - on préfère donner la mort
10. Randall Collins, Violence: a microsociological theory. Princeton: Princeton University Press, 2008.

11. Jack Katz, How emotions work. Chicago: University of Chicago Press, 1999.

12. J'ai eu l'occasion de débattre avec Randall Collins à deux reprises sur ce qui sépare sa démarche de la mienne, oralement, lors du Congrès de l'Association Internationale de Sociologie en 2010,

à Götebörg, et par écrit dans la revue Sociologica (2/2011), en réponse à son article, où il prenait une position critique par rapport à mes travaux:

«The invention and diffusion of social techniques of violence ».

13. Randall Collins, Interaction ritual chains. Princeton: Princeton University Press, 2004.

14. Samuel L. A. Marshall. Men against fire: the problem of battle command. New York: William Morrow \& Co., 1947. 
de loin que de près -, et donc sur la dépersonnalisation des victimes - on évite la confrontation psychologique, le face-à-face.

Randall Collins nous invite à être précis, et il a raison. Il note par exemple que si l'on observe de près les documents relatifs à un épisode de violence, on fait des constats qui éloignent des idées reçues. Il insiste sur l'importance de la domination émotionnelle, qui précéderait la destruction physique. II a aussi certainement raison de dire que la violence n'est pas chose facile, et qu'au contraire, il est difficile de la mettre en œuvre.

Randall Collins a bien conscience qu'une approche microsociologique n'épuise pas la compréhension de la violence, et il annonce un ouvrage qui permettra de passer à une approche macrosociologique. Mais pour l'instant, nous devons nous en tenir à son interactionnisme pragmatiste.

Peut-on réduire la violence à un « interactional accomplishment » en situation où l'émotion domine? Peut-on accepter en définitive l'idée que ce qui prime est la situation, et non les acteurs?

On notera, tout d'abord, qu'il n'est pas difficile de trouver des situations ou des expériences historiques qui démentent les affirmations de Randall, ou en tous cas qui mettent à mal toute prétention à la généralité ou à la validité théorique. Par exemple, comment rendre compte des expériences extrêmes où ce sont les plus proches, les voisins, ceux avec qui on a joué au football par exemple, qui sont l'objet des pires déchaînements violents, comme dans les purifications ethniques au Rwanda ou en ex-Yougoslavie? Dans ce dernier cas, il n'y a pas eu que des "snipers », tirant tout en étant invisibles, et dont Randall Collins fait grand cas, mais aussi des meurtriers s'en prenant à des gens qui les voyaient et les connaissaient.

Mais surtout, ce qui frappe, dans l'approche développée par Randall Collins, c'est la minoration du sens, ou sa disparition: les acteurs ne se comprennent qu'en situation, ils ne sont déterminés que dans et par l'interaction, sans laquelle la violence n’a pas lieu d'être. L'intersubjectivité ici est reine, sans qu'il soit nécessaire ou utile de s'intéresser à la subjectivité.

Un exemple précis va nous permettre de montrer en quoi un tel positionnement intellectuel est insuffisant, en tout cas s'il s'agit de proposer une théorie générale de la violence. 


\section{La mort de Clément Méric}

Cet exemple devrait être favorable à Randall Collins, tant la mort d'un jeune homme dont il va être question procède effectivement d'une interaction. Du moins en apparence. Le 6 juin 2013, un jeune militant d'extrême gauche, Clément Méric, meurt à Paris des suites d'une bagarre, la veille, avec des skinheads. II faut immédiatement écarter une explication par la crise économique et sociale, le chômage ou les difficultés des jeunes des quartiers populaires: I'affaire a lieu au cœur de Paris, dans un quartier agréable, et plus précisément après que Clément Méric et quelques amis aient croisé les skinheads dans un appartement privé où avait lieu une vente de vêtements d'une marque prisée aussi bien des militants d'extrême gauche que d'extrême droite. Les skinheads et les jeunes d'extrême gauche ne s'attendaient pas à se rencontrer dans cette vente, il n'y a pas là un quelconque rendez-vous plus ou moins organisé entre bandes rivales politiquement, mais le fruit d'un hasard. II semble que ce soient les jeunes d'extrême gauche qui ont commencé à agresser verbalement et à provoquer les skinheads, et qui leur ont proposé de se retrouver dehors. Et là, les coups sont partis, et un skinhead a tué Clément Méric d’un ou deux coups de poing.

Il est donc en première instance tout à fait utile de se référer à l'interactionnisme pragmatiste que propose Randall Collins: une situation, de surcroît imprévue, a généré une altercation verbale, qui s'est ensuivie d'une bagarre qui a mal tourné. La mort n'était ni prévue, ni voulue, elle est accidentelle.

Certes. Mais regardons de plus près les auteurs de la violence. Pour avoir étudié il y a un quart de siècle un groupe de skinheads français ${ }^{15}$ (de surcroît dont le leader est devenu le principal dirigeant des organisations d'extrême droite qui donnent un sens idéologique à ce type d'acteurs), je peux affirmer qu'ils sont plus soucieux de préparation physique à la violence, que de préparation politique ou idéologique. En général, ils s'entraînent au combat, et s'alimentent en conséquence. Dans la recherche que j'ai conduite, et qui a consisté à faire rencontrer à un groupe d'une douzaine de skinheads une série d'interlocuteurs avant de leur proposer de discuter de mon analyse de leur action, j'ai passé une cinquantaine d'heures avec eux, et ils étaient très soucieux, si j'excepte leur leader, d'être en condition physique pour l'action violente - ce qui ne les empêchait pas de consommer beaucoup de bière. Je suppose que le meurtrier de Clément Méric et ses amis n'échappent guère à cette description, ou accessoirement.

La mort de Clément Méric est accidentelle, certes, mais l'accident n'aurait pas eu lieu si les skinheads n'avaient pas été des acteurs prêts à passer à une violence physique considérable, et déterminés à le faire à l'occasion, ou si nécessaire. L'inte-
15. Cf. Michel Wieviorka et alii, La France raciste. Paris: Seuil, 1992 (et plus particulièrement le chapitre 10). 
raction doit beaucoup au hasard, certes mais celui-ci n'est pas seul en cause: ainsi, la vente privée à laquelle les participants au drame s'étaient rendus visait, entre autres, un public bien précis, extrémiste.

La droite politique française, et même le Front national, où il faut plutôt voir une droite radicale ou extrémiste, se sont nettement démarqués des skinheads, et l'interprétation dominante, à gauche, sitôt la nouvelle connue, a consisté à minimiser les dimensions d'interaction du drame, pour brandir le spectre de la montée de l'extrême droite, proposer des comparaisons avec 1934 - période de grandes inquiétudes politiques relatives au fascisme - et pour demander, ce qui fut suivi d'effet quelques jours plus tard, l'interdiction des organisations d'extrême droite qui, pourtant de très loin, ont pu inspirer idéologiquement le meurtrier. II fallait au contraire, et je m'y suis personnellement employé dans plusieurs interventions dans les médias, mettre en lumière la réalité des faits, et leur caractère d'interaction ayant mal tourné - ce qui pourrait donner raison à Randall Collins. Mais ces faits n'auraient pas existé si, en amont de la situation ou du moment, les acteurs n’avaient pas été largement préparés, subjectivement, mais aussi physiquement, à passer à l'acte. La mort est accidentelle et relève de l'interaction, la violence, elle, s'explique autrement.

Et l'interaction elle-même n'a eu lieu que parce que deux subjectivités s'opposaient et se sont heurtées, d'abord sous la forme d'agressions verbales et de provocations, ensuite sous celle de la violence, avec, pour au moins l'une d'entre elle, une forte propension à se transcrire de la sorte. C'est pourquoi, tout en reconnaissant l'utilité d'une approche s'intéressant à la situation et aux interactions qu'elle autorise, il me semble préférable, pour approfondir la compréhension de la violence, d'envisager une deuxième piste, profondément différente s'il s'agit de ses bases théoriques: la piste du sujet, de la subjectivité et, mieux encore, des processus de subjectivation et de désubjectivation.

\section{Le sujet et la violence}

Éloignons-nous, par conséquent, des approches qui privilégient l'interaction et donc l'intersubjectivité, pour nous intéresser à la subjectivité de celui qui passe à la violence, et donc au sens qu'elle charrie, quitte à ce que ce soit un sens étrange, perverti par rapport à des significations sociales, politiques ou culturelles plus banales. Ce qui implique d'en préciser le concept, et ceux qui en sont proches - sujet, subjectivation, désubjectivation.

Nous procéderons à cet effort en deux temps. 
Une première étape dans la réflexion théorique consiste à adopter une perspective centrée sur le concept de sujet.

Celui-ci a été relancé dans les sciences humaines et sociales après une longue période d'un purgatoire dominé par diverses variantes d'un structuralisme pourchassant le sujet et prônant sa mort. Déjà Michel Foucault, le « dernier Foucault », celui de La volonté de savoir et non celui de Les mots et les choses, installait le sujet au cœur de sa pensée. Depuis, la sociologie, l'anthropologie, l'histoire, les sciences politiques ont développé, en liaison avec la philosophie, de nombreuses recherches qui s'appuient le plus souvent sur une conception positive, presque romantique du " sujet ». Celui-ci, par exemple chez Alain Touraine ou Hans Joas ${ }^{16}$, est défini par sa capacité d'agir, il renvoie à des images de liberté, de maîtrise de soi, de capacité de choisir, de décider, de contrôler son expérience. Il est responsable, doté d'un sens de la collectivité, car pour pouvoir être sujet soi-même, il faut admettre que les autres humains puissent bénéficier de la même possibilité. Le « sujet » des sciences humaines et sociales contemporaines est au plus loin de l'assujettissement, qui est synonyme de soumission à une domination, il est affirmation de liberté et de responsabilité. Le retour du sujet s'est opéré avec l'idée que ce dernier s'écarte de l'assujettissement, c'est-à-dire d'une relation de subordination et d'appartenance à un pouvoir qui le prive de réflexion et d'autonomie; le sujet contemporain met en cause le pouvoir, résiste aux normes et aux règles, il existe en soi, et pour soi - il relève du « souci de soi » dont parle Michel Foucault ${ }^{17}$.

Tout n'est pas toujours parfaitement clair dans les propositions théoriques qui soustendent cette image du sujet, dont on ne sait pas nécessairement s'il est une virtualité qui préexiste à l'action, ou s'il se constitue dans l'action, dans l'expérience. Le sujet des sciences sociales est parfois une qualité anthropologique relativement abstraite ou indéterminée, qui se transforme, ou non, en action, il est parfois, différemment une qualité sociale, civique, culturelle, ou religieuse, c'est par exemple un ouvrier doté de conscience de classe, un citoyen, ou bien encore un individu appartenant à une communauté culturelle, ou religieuse, il est alors déterminé, ancré dans du réel, du concret. Toujours est-il que le sujet est ici liberté, émancipation, résistance, construction et affirmation de soi.

Mais s'il s'agit de violence, d'autres questions méritent d'être soulevées: peut-on utiliser un concept de sujet pour aborder le mal - la barbarie, le vol, le viol, le terrorisme, le crime? En fait, oui, à condition de proposer un concept renouvelé de sujet, qui tienne compte, précisément, du caractère connoté négativement de la violence. C'est pourquoi, dans un ouvrage sur la violence ${ }^{18}$, j'ai développé une conceptualisation qui repose sur plusieurs types principaux de sujet et notamment:
16. Cf., par exemple, François Dubet et Michel Wieviorka (Dir.), Penser le sujet. Autour d'Alain Touraine. Paris: Fayard, 1995; et Hans Joas, La créativité de l'agir. Paris: Cerf, 2001 [1992]

17. Michel Foucault, Histoire de la sexualité, v. 3, "Le souci de soi ». Paris: Gallimard, 2001.

18. Michel Wieviorka Violence: a new approach. London: Sage, 2009 [2006]. 
- «Sujet flottant »-c'est le sujet qui devient violent parce qu'il ne peut pas devenir acteur du fait de conditions particulièrement défavorables: exclusion, racisme, impossibilité de faire valoir des demandes sociales par exemple.

- L'hyper-sujet, comme le sujet flottant, ne peut pas devenir acteur du fait de conditions sociales, économiques, culturelles ou politiques qui l'en empêchent. Mais le passage à la violence est précédé, ou accompagné par un processus de recherche du sens, par une surcharge de sens qui peut prendre l'allure de l'idéologie ou de la religion. La violence est alors indissociable d'une construction intellectuelle qui aboutit à la légitimer et lui confère une force particulière. C'est ainsi que des variantes particulièrement radicales de l'islam peuvent apporter son sens à une action qui devient possible sous la forme d'une violence extrême, destructrice et, dans le cas du martyrisme islamiste, auto-destructrice.

- Le non-sujet agit de manière violente tout en se disant non responsable, sans s'engager apparemment comme Sujet. Par exemple, il dit obéir à une autorité légitime, comme Adolphe Eichmann, qui prétendait qu'il aurait même tué son père si Hitler le lui avait demandé.

- L'anti-sujet agit violemment par plaisir, il pratique la violence pure, la violence pour la violence, il déshumanise sa victime, agit par cruauté, par sadisme, comme les militaires de l'armée américaine dans le centre de détention d'Abou Graïd.

19. Je traite de cet enjeu dans Michel Wieviorka, Evil. London: Polity Press, 2012 (et plus particulièrement dans le chapitre 2, "An end to violence »).
Avec cette esquisse de typologie, que je ne fais ici qu'évoquer, on commence à avoir un concept de sujet un peu plus élaboré. À partir de là, nous pouvons réfléchir à la délicate question de la sortie de la violence: chaque type de sujet, en effet, appelle des réponses différentes ${ }^{19}$. Face à l'anti-sujet, par exemple, seule la répression peut être efficace, alors que la violence du sujet flottant peut être combattue par des politiques sociales ou culturelles, ou bien encore en transformant en débats et en conflit institutionnalisé les demandes non satisfaites.

Concrètement, les figures du sujet violent s'entremêlent, ou se succèdent, ou bien encore s'encapsulent l'une dans l'autre, ce qui ne peut que compliquer l'action de ceux qui veulent agir contre la violence. Et surtout, la violence est vite instable, c'est un phénomène toujours susceptible d'évolution, toujours difficile, pour ses acteurs, à contrôler et à maintenir à un niveau voulu pour des raisons stratégiques, instrumentales: la violence a tôt fait de s'emballer, d'échapper au contrôle. C'est pourquoi les acteurs de la violence donnent dans certaines expériences l'image de profondes transformations, par exemple entre les premiers moments timides, mesurés, d'une 
action apparemment contrôlée, et le déchaînement furieux, barbare, dépourvu de toute humanité, d'une violence sans limites.

Le sujet de la violence n'est donc pas lui-même une figure nécessairement stabilisée, contrôlée, il peut par exemple être au départ flottant et non meurtrier, juste émeutier pendant une nuit par exemple, pour devenir radical, un hyper-sujet porté par une haine inextinguible et débouchant sur une violence extrême au sein de laquelle la cruauté peut faire son apparition. C'est pourquoi le concept de sujet, même amélioré comme nous le proposons, est insuffisant. Si le protagoniste de la violence entre dans celle-ci, puis lui donne éventuellement un sens et des expressions changeantes, alors il faut s'intéresser non pas à l'idée d'un sujet défini une fois pour toutes, mais à celle de processus dans lesquels changent le sens mis ou contenu dans la violence, et la subjectivité de l'acteur.

\section{Subjectivation, désubjectivation}

Passer du sujet et de la subjectivité, aux processus de la subjectivation et la désubjectivation, c'est donc adopter une forme de raisonnement différente, qui évite toute essentialisation ou naturalisation du "sujet », et qui permet de sortir des questionnements relatifs au statut même du sujet, dont on a vu qu'il est problématique, puisqu'il peut ou bien être défini comme en amont de toute action ou expérience, ou bien être conçu comme indissociable de la pratique.

Ces processus sont de deux ordres, ils relèvent de deux types de logiques opposées, et qui peuvent néanmoins être co-présentes. D'une part, en effet, la violence peut être produite au fil de processus de subjectivation. C'est ainsi, par exemple, que bien des jeunes habitant un quartier populaire en France ou au Royaume-Uni disent, après leur première expérience d'une émeute urbaine, que celle-ci leur avait permis de donner un sens à leur existence, qu'elle a eu un rôle fondateur à partir duquel ils réexaminent leur mode de vie, décident d'entrer en religion, ou dans une association musicale, ou sportive - parfois aussi entrent dans la spirale de la délinquance, ou d'une violence sans cesse plus poussée. Le thème de la violence fondatrice, généralement développé dans l'anthropologie des communautés par des auteurs comme René Girard ${ }^{20}$ peut ainsi être associé à la compréhension de processus de subjectivation centré non pas sur le groupe, mais sur des personnes singulières.

Mais d'autre part, et beaucoup plus largement, la violence se développe en même temps que le sens de l'action se perd, se pervertit, se déplace pour faire de l'acteur, de plus en plus, un individu ou un groupe se rapprochant de la violence pure, la violence pour la violence, s'enfermant dans la spirale de la perte du sens, ou de son

20. René Girard, La violence et le sacré Paris: Hachette, 1972 
remplacement par une idéologie ou une religion radicalisée. La logique est alors celle de la désubjectivation.

Là encore, les réponses à la violence varient, selon qu'il s'agit d'une subjectivation provisoirement assurée à travers la violence, ou d'une désubjectivation plus ou moins poussée. S'il s'agit d'une logique provisoire de subjectivation, alors, l'action contre la violence doit consister à encourager l'inflexion de cette subjectivation de sorte qu'elle se dirige, après le moment fondateur, vers d'autres formes d'action, éventuellement inscrites dans une conflictualisation institutionnalisée, alors que s'il s'agit d'une logique, plus courante, de désubjectivation, le retour au sens n'est possible que si cette logique n'est pas encore trop poussée, faute de quoi seule la répression peut s'avérer efficace. Dans tous les cas, le fait de penser en termes de processus implique, pour qui veut réfléchir à l'action contre la violence, de définir les modalités permettant d'enrayer le processus, le plus tôt possible.

Ainsi peut s'ébaucher un nouveau paradigme de la violence, né de l'épuisement des modes d'approche les plus classiques, de la critique des démarches interactionnistes et de la prise en compte d'un concept de sujet lui-même prolongé ou dépassé par l'idée de processus de subjectivation et de désubjectivation.

Étant donné l'existence de différents types de violence et, surtout, en mettant en relief la violence collective, cet article présente un bref examen de quelques théories sociologiques au sujet de la violence, considérées à partir de trois paradigmes classiques, y inclus les analyses qui privilégient la dimension instrumentale, celles qui voient dans la violence une réponse ou une réaction a des situations de crise et, à la fin, celles qui insistent sur l'idée d'une culture de violence. Les trois paradigmes renferment un biais déterministe qui fait obstacle à que les sciences sociales soient bien préparées afin de montrer les conditions qui rendent la violence possible. Cet article met en relief aussi l'importance d'éviter le psychologisme et le sociologisme lorsque l'on fait l'analyse de la violence. II présente deux orientations différentes: dans la première, il discute l'interaccionisme - y inclus l'intersubjectivité - et montre quelques aspects positifs, surtout, quelques limites; dans la seconde, II met en relief l'orientation théorique centrée dans le sujet, dans la subjectivité et, surtout, dans le processus de subjectivation et de désubjectivation. II postule le besoin de définir les concepts de subjectivité, de sujet, de subjectivation et de désubjectivation et il définit, au moyen de ce propos, quatre types de sujet qui peuvent même coexister dans un seul acteur. II explicite, à la fin, l'insuffisance du concept de sujet et II propose passer, dans cette analyse, de l'évidence sur le sujet et sur la subjectivation à l'évidence sur les processus de subjectivation et de désubjecvation et, par conséquence, éviter l'essencialisation ou la naturalisation du sujet.

Mots-clés: violence, sujet, subjectivité, subjectivation, désubjectivation.

Abstract: This paper examines briefly some sociological theories about violence, taking into account that there are different types of violence, and focusing specifically on collective violence. The analysis departs from three classic paradigms: the instrumental dimension; the interpretation of violence as an answer or a reaction to some situation, especially a crisis; and finally those studies that insist on the idea of a culture of violence. These three paradigms contain a deterministic bias which postulates a causality or a collection of causes to explain violence, thus making it difficult for the social sciences to clarify the conditions that make violence possible. The paper also emphasizes the importance 
of avoiding "psychologisms" and "sociologsms" in the analysis of violence, introducing instead two other perspectives: in the first one, interactionism is discussed - with the intersubjectivity component - indicating some of its positive aspects, and, above all its limits; the focus of the second one is a theoretical view centered on the subject (actor, individual), the subjectivity and the desubjectivity. The need for a definition of the concepts of subjectivity, subject, subjectivation and desubjectivation is postulated, whilst four types of subject hat may co-exist within the same actor are proposed. Finally, the paper shows the shortcoming of the concept of subject and proposes an analysis based instead on the subjectivity and the subjectivation and desubjectivation processes, avoiding the essentialization or naturalization of the subject.

Keywords: violence, subject, subjectivity, subjectivation, desubjectivation. 